\title{
Observation of the Leptonic Decay $D^{+} \rightarrow \tau^{+} \nu_{\tau}$
}

M. Ablikim, ${ }^{1}$ M. N. Achasov, ${ }^{10, d}$ P. Adlarson ${ }^{59}$ S. Ahmed,${ }^{15}$ M. Albrecht, ${ }^{4}$ M. Alekseev ${ }^{58 a, 58 c}$ A. Amoroso, ${ }^{58 a, 58 c}$ F. F. An, ${ }^{1}$ Q. An ${ }^{55,43}$ Y. Bai, ${ }^{42}$ O. Bakina, ${ }^{27}$ R. Baldini Ferroli, ${ }^{23 a}$ I. Balossino, ${ }^{24 a}$ Y. Ban, ${ }^{35}$ K. Begzsuren, ${ }^{25}$ J. V. Bennett, ${ }^{5}$ N. Berger, ${ }^{26}$ M. Bertani, ${ }^{23 a}$ D. Bettoni, ${ }^{24 a}$ F. Bianchi, ${ }^{58 a, 58 c}$ J. Biernat, ${ }^{59}$ J. Bloms, ${ }^{52}$ I. Boyko, ${ }^{27}$ R. A. Briere, ${ }^{5}$ H. Cai,${ }^{60}$ X. Cai, ${ }^{1,43}$ A. Calcaterra, ${ }^{23 a}$ G. F. Cao, ${ }^{1,47}$ N. Cao, ${ }^{1,47}$ S. A. Cetin, ${ }^{46 b}$ J. Chai,${ }^{58 c}$ J. F. Chang, ${ }^{1,43}$ W. L. Chang, ${ }^{1,47}$ G. Chelkov, ${ }^{27, b, c}$ D. Y. Chen, ${ }^{6}$ G. Chen, ${ }^{1}$ H. S. Chen, ${ }^{1,47}$ J. C. Chen, ${ }^{1}$ M. L. Chen, ${ }^{1,43}$ S. J. Chen, ${ }^{33}$ Y. B. Chen, ${ }^{1,43}$ W. Cheng, ${ }^{58 c}$ G. Cibinetto, ${ }^{24 a}$ F. Cossio, ${ }^{58 c}$ X. F. Cui, ${ }^{34}$ H. L. Dai, ${ }^{1,43}$ J. P. Dai, ${ }^{38, h}$ X. C. Dai, ${ }^{1,47}$ A. Dbeyssi, ${ }^{15}$ D. Dedovich,${ }^{27}$ Z. Y. Deng, ${ }^{1}$ A. Denig, ${ }^{26}$ I. Denysenko, ${ }^{27}$ M. Destefanis, ${ }^{58 a, 58 c}$ F. De Mori ${ }^{58 a, 58 c}$ Y. Ding, ${ }^{31}$ C. Dong, ${ }^{34}$ J. Dong, ${ }^{1,43}$ L. Y. Dong,, 47 M. Y. Dong, ${ }^{1,43,47}$ Z. L. Dou, ${ }^{33}$ S. X. Du, ${ }^{63}$ J. Z. Fan, ${ }^{45}$ J. Fang, ${ }^{1,43}$ S. S. Fang, ${ }^{1,47}$ Y. Fang, ${ }^{1}$ R. Farinelli, ${ }^{24 a, 24 b}$ L. Fava, ${ }^{58 b, 58 c}$ F. Feldbauer, ${ }^{4}$ G. Felici, ${ }^{23 a}$ C. Q. Feng, ${ }^{55,43}$ M. Fritsch, ${ }^{4}$ C. D. Fu, ${ }^{1}$ Y. Fu, ${ }^{1}$ Q. Gao, ${ }^{1}$ X. L. Gao, ${ }^{55,43}$ Y. Gao, ${ }^{45}$ Y. Gao,${ }^{56}$ Y. G. Gao, ${ }^{6}$ Z. Gao, ${ }^{55,43}$ B. Garillon, ${ }^{26}$ I. Garzia, ${ }^{24 a}$ E. M. Gersabeck, ${ }^{50}$ A. Gilman, ${ }^{51}$ K. Goetzen, ${ }^{11}$ L. Gong, ${ }^{34}$ W. X. Gong, ${ }^{1,43}$ W. Gradl, ${ }^{26}$ M. Greco, ${ }^{58 a, 58 c}$ L. M. Gu, ${ }^{33}$ M. H. Gu, ${ }^{1,43}$ S. Gu, ${ }^{2}$ Y. T. Gu, ${ }^{13}$ A. Q. Guo, ${ }^{22}$ L. B. Guo, ${ }^{32}$ R. P. Guo ${ }^{36}$ Y. P. Guo, ${ }^{26}$ A. Guskov, ${ }^{27}$ S. Han,${ }^{60}$ X. Q. Hao, ${ }^{16}$ F. A. Harris, ${ }^{48}$ K. L. He, ${ }^{1,47}$ F. H. Heinsius, ${ }^{4}$ T. Held, ${ }^{4}$

Y. K. Heng, ${ }^{1,43,47}$ M. Himmelreich, ${ }^{11, g}$ Y. R. Hou, ${ }^{47}$ Z. L. Hou, ${ }^{1}$ H. M. Hu ${ }^{1,47}$ J. F. Hu, ${ }^{38, h}$ T. Hu, ${ }^{1,43,47}$ Y. Hu, ${ }^{1}$ G. S. Huang, ${ }^{5,43}$ J. S. Huang, ${ }^{16}$ X. T. Huang,${ }^{37}$ X. Z. Huang, ${ }^{33}$ N. Huesken, ${ }^{52}$ T. Hussain, ${ }^{57}$ W. Ikegami Andersson, ${ }^{59}$ W. Imoehl, ${ }^{22}$ M. Irshad ${ }^{55,43}$ Q. Ji, ${ }^{1}$ Q. P. Ji, ${ }^{16}$ X. B. Ji, ${ }^{1,47}$ X. L. Ji, ${ }^{1,43}$ H. L. Jiang, ${ }^{37}$ X. S. Jiang, ${ }^{1,43,47}$ X. Y. Jiang, ${ }^{34}$ J. B. Jiao, ${ }^{37}$ Z. Jiao, ${ }^{18}$ D. P. Jin, ${ }^{1,43,47}$ S. Jin, ${ }^{33}$ Y. Jin, ${ }^{49}$ T. Johansson, ${ }^{59}$ N. Kalantar-Nayestanaki, ${ }^{29}$ X. S. Kang, ${ }^{31}$ R. Kappert, ${ }^{29}$ M. Kavatsyuk, ${ }^{29}$ B. C. Ke, ${ }^{1}$ I. K. Keshk, ${ }^{4}$ A. Khoukaz, ${ }^{52}$ P. Kiese, ${ }^{26}$ R. Kiuchi, ${ }^{1}$ R. Kliemt, ${ }^{11}$ L. Koch, ${ }^{28}$ O. B. Kolcu, ${ }^{46 b, f}$ B. Kopf ${ }^{4}$ M. Kuemmel, ${ }^{4}$ M. Kuessner, ${ }^{4}$ A. Kupsc, ${ }^{59}$ M. Kurth, ${ }^{1}$ M. G. Kurth, ${ }^{1,47}$ W. Kühn, ${ }^{28}$ J. S. Lange, ${ }^{28}$ P. Larin, ${ }^{15}$ L. Lavezzi, ${ }^{58 c}$ H. Leithoff, ${ }^{26}$ T. Lenz, ${ }^{26}$ C. Li ${ }^{59}$ Cheng Li,${ }^{55,43}$ D. M. Li,${ }^{63}$ F. Li, ${ }^{1,43}$ F. Y. Li,${ }^{35}$ G. Li, ${ }^{1}$ H. B. Li, ${ }^{1,47}$ H. J. Li ${ }^{9, j}$ J. C. Li, ${ }^{1}$ J. W. Li, ${ }^{41}$ Ke Li, ${ }^{1}$ L. K. Li, ${ }^{1}$ Lei Li, ${ }^{3}$ P. L. Li, ${ }^{55,43}$ P. R. Li, ${ }^{30}$ Q. Y. Li, ${ }^{37}$ W. D. Li, ${ }^{1,47}$ W. G. Li, ${ }^{1}$ X. H. Li ${ }^{55,43}$ X. L. Li,${ }^{37}$ X. N. Li, ${ }^{1,43}$ Z. B. Li, ${ }^{44}$ Z. Y. Li ${ }^{44}$ H. Liang, ${ }^{55,43}$ H. Liang, ${ }^{1,47}$ Y. F. Liang, ${ }^{40}$ Y. T. Liang, ${ }^{28}$ G. R. Liao, ${ }^{12}$ L. Z. Liao, ${ }^{1,47}$ J. Libby, ${ }^{21}$ C. X. Lin, ${ }^{44}$ D. X. Lin, ${ }^{15}$ Y. J. Lin, ${ }^{13}$ B. Liu, ${ }^{38, h}$ B. J. Liu, ${ }^{1}$ C. X. Liu, ${ }^{1}$ D. Liu, ${ }^{55,43}$ D. Y. Liu, ${ }^{38, h}$ F. H. Liu,${ }^{39}$ Fang Liu, ${ }^{1}$ Feng Liu, ${ }^{6}$ H. B. Liu, ${ }^{13}$ H. M. Liu, ${ }^{1,47}$ Huanhuan Liu, ${ }^{1}$ Huihui Liu, ${ }^{17}$ J. B. Liu, ${ }^{55,43}$ J. Y. Liu, ${ }^{1,47}$ K. Y. Liu, ${ }^{31}$ Ke Liu, ${ }^{6}$ L. Y. Liu, ${ }^{13}$ Q. Liu, ${ }^{47}$ S. B. Liu, ${ }^{55,43}$ T. Liu, ${ }^{1,47}$ X. Liu, ${ }^{30}$ X. Y. Liu, ${ }^{1,47}$ Y. B. Liu, ${ }^{34}$ Z. A. Liu, ${ }^{1,43,47}$ Zhiqing Liu, ${ }^{37}$ Y. F. Long, ${ }^{35}$ X. C. Lou, ${ }^{1,43,47}$ H. J. Lu, ${ }^{18}$ J. D. Lu, ${ }^{1,47}$ J. G. Lu, ${ }^{1,43}$ Y. Lu, ${ }^{1}$ Y. P. Lu, ${ }^{1,43}$ C. L. Luo, ${ }^{32}$ M. X. Luo, ${ }^{62}$ P. W. Luo, ${ }^{44}$ T. Luo, ${ }^{9, j}$ X. L. Luo, ${ }^{1,43}$ S. Lusso, ${ }^{58 c}$ X. R. Lyu, ${ }^{47}$ F. C. Ma, ${ }^{31}$ H. L. Ma, ${ }^{1}$ L. L. Ma, ${ }^{37}$ M. M. Ma, ${ }^{1,47}$ Q. M. Ma, ${ }^{1}$ X. N. Ma ${ }^{34}$ X. X. Ma,${ }^{1,47}$ X. Y. Ma, ${ }^{1,43}$ Y. M. Ma,${ }^{37}$ F. E. Maas, ${ }^{15}$ M. Maggiora, ${ }^{58 a, 58 c}$ S. Maldaner, ${ }^{26}$ S. Malde, ${ }^{53}$ Q. A. Malik,${ }^{57}$ A. Mangoni, ${ }^{23 b}$ Y. J. Mao, ${ }^{35}$ Z. P. Mao, ${ }^{1}$ S. Marcello, ${ }^{58 a, 58 c}$ Z. X. Meng, ${ }^{49}$ J. G. Messchendorp, ${ }^{29}$ G. Mezzadri, ${ }^{24 a}$ J. Min, ${ }^{1,43}$ T. J. Min, ${ }^{33}$ R. E. Mitchell, ${ }^{22}$ X. H. Mo, ${ }^{1,43,47}$ Y. J. Mo, ${ }^{6}$

C. Morales Morales,${ }^{15}$ N. Yu. Muchnoi, ${ }^{10, d}$ H. Muramatsu, ${ }^{51}$ A. Mustafa, ${ }^{4}$ S. Nakhoul,${ }^{11, g}$ Y. Nefedov,${ }^{27}$ F. Nerling, ${ }^{11, g}$ I. B. Nikolaev, ${ }^{10, \mathrm{~d}}$ Z. Ning, ${ }^{1,43}$ S. Nisar, ${ }^{8, \mathrm{k}}$ S. L. Niu, ${ }^{1,43}$ S. L. Olsen, ${ }^{47}$ Q. Ouyang, ${ }^{1,43,47}$ S. Pacetti, ${ }^{23 \mathrm{~b}}$ Y. Pan, ${ }^{55,43}$ M. Papenbrock, ${ }^{59}$ P. Patteri, ${ }^{23 a}$ M. Pelizaeus, ${ }^{4}$ H. P. Peng,${ }^{55,43}$ K. Peters, ${ }^{11,9}$ J. Pettersson, ${ }^{59}$ J. L. Ping, ${ }^{32}$ R. G. Ping, ${ }^{1,47}$ A. Pitka, ${ }^{4}$ R. Poling, ${ }^{51}$ V. Prasad,${ }^{55,43}$ H. R. Qi ${ }^{2}$ M. Qi,${ }^{33}$ T. Y. Qi, ${ }^{2}$ S. Qian, ${ }^{1,43}$ C. F. Qiao, ${ }^{47}$ N. Qin, ${ }^{60}$ X. P. Qin, ${ }^{13}$ X. S. Qin, ${ }^{4}$ Z. H. Qin, ${ }^{1,43}$ J. F. Qiu, ${ }^{1}$ S. Q. Qu ${ }^{34}$ K. H. Rashid,${ }^{57, i}$ K. Ravindran, ${ }^{21}$ C. F. Redmer, ${ }^{26}$ M. Richter, ${ }^{4}$ A. Rivetti,${ }^{58 c}$ V. Rodin, ${ }^{29}$ M. Rolo, ${ }^{58 c}$ G. Rong, ${ }^{1,47}$ Ch. Rosner,${ }^{15}$ M. Rump ${ }^{52}$ A. Sarantsev, ${ }^{27, e}$ M. Savriée, ${ }^{24 b}$ Y. Schelhaas, ${ }^{26}$ K. Schoenning, ${ }^{59}$ W. Shan, ${ }^{19}$ X. Y. Shan, ${ }^{55,43}$ M. Shao, ${ }^{55,43}$ C. P. Shen, ${ }^{2}$ P. X. Shen, ${ }^{34}$ X. Y. Shen, ${ }^{1,47}$ H. Y. Sheng, ${ }^{1}$ X. Shi, ${ }^{1,43}$ X. D. Shi, ${ }^{55,43}$ J. J. Song, ${ }^{37}$ Q. Q. Song, ${ }^{55,43}$ X. Y. Song, ${ }^{1}$ S. Sosio,${ }^{58 a, 58 c}$ C. Sowa, ${ }^{4}$ S. Spataro, ${ }^{58 a, 58 c}$ F. F. Sui, ${ }^{37}$ G. X. Sun, ${ }^{1}$ J. F. Sun ${ }^{16}$ L. Sun, ${ }^{60}$ S. S. Sun, ${ }^{1,47}$ X. H. Sun, ${ }^{1}$ Y. J. Sun, ${ }^{55,43}$ Y. K. Sun, ${ }^{55,43}$ Y.Z. Sun, ${ }^{1}$ Z. J. Sun, ${ }^{1,43}$ Z. T. Sun, ${ }^{1}$ Y. T. $\operatorname{Tan}^{55,43}$ C. J. Tang, ${ }^{40}$ G. Y. Tang, ${ }^{1}$ X. Tang, ${ }^{1}$ V. Thoren, ${ }^{59}$ B. Tsednee, ${ }^{25}$ I. Uman, ${ }^{46 d}$ B. Wang, ${ }^{1}$ B. L. Wang, ${ }^{47}$ C. W. Wang, ${ }^{33}$

D. Y. Wang, ${ }^{35}$ K. Wang, ${ }^{1,43}$ L. L. Wang, ${ }^{1}$ L. S. Wang, ${ }^{1}$ M. Wang, ${ }^{37}$ M. Z. Wang ${ }^{35}$ Meng Wang, ${ }^{1,47}$ P. L. Wang, ${ }^{1}$

R. M. Wang, ${ }^{61}$ W. P. Wang, ${ }^{55,43}$ X. Wang, ${ }^{35}$ X. F. Wang, ${ }^{1}$ X. L. Wang, ${ }^{9, j}$ Y. Wang, ${ }^{55,43}$ Y. Wang, ${ }^{44}$ Y. F. Wang, ${ }^{1,43,47}$ Z. Wang, ${ }^{1,43}$ Z. G. Wang, ${ }^{1,43}$ Z. Y. Wang, ${ }^{1}$ Zongyuan Wang, ${ }^{1,47}$ T. Weber, ${ }^{4}$ D. H. Wei, ${ }^{12}$ P. Weidenkaff, ${ }^{26}$ H. W. Wen, ${ }^{32}$ S. P. Wen, ${ }^{1}$ U. Wiedner, ${ }^{4}$ G. Wilkinson, ${ }^{53}$ M. Wolke, ${ }^{59}$ L. H. Wu, ${ }^{1}$ L. J. Wu,${ }^{1,47}$ Z. Wu, ${ }^{1,43}$ L. Xia, ${ }^{55,43}$ Y. Xia, ${ }^{20}$ S. Y. Xiao, ${ }^{1}$ Y. J. Xiao, ${ }^{1,47}$ Z. J. Xiao, ${ }^{32}$ Y. G. Xie, ${ }^{1,43}$ Y. H. Xie, ${ }^{6}$ T. Y. Xing, ${ }^{1,47}$ X. A. Xiong, ${ }^{1,47}$ Q. L. Xiu, ${ }^{1,43}$ G. F. Xu, ${ }^{1}$ J. J. Xu, ${ }^{33}$ L. Xu, ${ }^{1}$ Q. J. Xu, ${ }^{14}$ W. Xu, ${ }^{1,47}$ X. P. Xu, ${ }^{41}$ F. Yan, ${ }^{56}$ L. Yan, ${ }^{58 a, 58 c}$ W. B. Yan, ${ }^{55,43}$ W. C. Yan, ${ }^{2}$ Y. H. Yan, ${ }^{20}$ H. J. Yang, ${ }^{38, h}$ H. X. Yang, ${ }^{1}$ L. Yang ${ }^{60}$ R. X. Yang, ${ }^{55,43}$ S. L. Yang, ${ }^{1,47}$ Y. H. Yang, ${ }^{33}$ Y. X. Yang, ${ }^{12}$ Yifan Yang, ${ }^{1,47}$ Z. Q. Yang, ${ }^{20}$ M. Ye, ${ }^{1,43}$ M. H. Ye, ${ }^{7}$ J. H. Yin, ${ }^{1}$ Z. Y. You, ${ }^{44}$ B. X. Yu, ${ }^{1,43,47}$ C. X. Yu, ${ }^{34}$ J. S. Yu, ${ }^{20}$ T. Yu, ${ }^{56}$ C. Z. Yuan, ${ }^{1,47}$ X. Q. Yuan, ${ }^{35}$ Y. Yuan, ${ }^{1}$ A. Yuncu, ${ }^{46 b, a}$ A. A. Zafar, ${ }^{57}$ Y. Zeng, ${ }^{20}$ B. X. Zhang, ${ }^{1}$ B. Y. Zhang, ${ }^{1,43}$ C. C. Zhang, ${ }^{1}$ D. H. Zhang, ${ }^{1}$ H. H. Zhang, ${ }^{44}$ 
H. Y. Zhang, ${ }^{1,43}$ J. Zhang, ${ }^{1,47}$ J. L. Zhang, ${ }^{61}$ J. Q. Zhang, ${ }^{4}$ J. W. Zhang, ${ }^{1,43,47}$ J. Y. Zhang, ${ }^{1}$ J. Z. Zhang, ${ }^{1,47}$ K. Zhang, ${ }^{1,47}$ L. Zhang, ${ }^{45}$ S. F. Zhang, ${ }^{33}$ T. J. Zhang, ${ }^{38, h}$ X. Y. Zhang, ${ }^{37}$ Y. Zhang, ${ }^{55,43}$ Y. H. Zhang, ${ }^{1,43}$ Y. T. Zhang, ${ }^{55,43}$ Yang Zhang, ${ }^{1}$ Yao Zhang, ${ }^{1}$ Yi Zhang, ${ }^{9, j}$ Yu Zhang, ${ }^{47}$ Z. H. Zhang, ${ }^{6}$ Z. P. Zhang, ${ }^{55}$ Z. Y. Zhang, ${ }^{60}$ G. Zhao, ${ }^{1}$ J. W. Zhao, ${ }^{1,43}$ J. Y. Zhao, ${ }^{1,47}$ J. Z. Zhao, ${ }^{1,43}$ Lei Zhao, ${ }^{55,43}$ Ling Zhao, ${ }^{1}$ M. G. Zhao, ${ }^{34}$ Q. Zhao, ${ }^{1}$ S. J. Zhao, ${ }^{63}$ T. C. Zhao, ${ }^{1}$ Y. B. Zhao, ${ }^{1,43}$ Z. G. Zhao ${ }^{55,43}$ A. Zhemchugov, ${ }^{27, b}$ B. Zheng, ${ }^{56}$ J. P. Zheng, ${ }^{1,43}$ Y. Zheng, ${ }^{35}$ Y. H. Zheng, ${ }^{47}$ B. Zhong, ${ }^{32}$ L. Zhou, ${ }^{1,43}$ L. P. Zhou, ${ }^{1,47}$ Q. Zhou, ${ }^{1,47}$ X. Zhou, ${ }^{60}$ X. K. Zhou, ${ }^{47}$ X. R. Zhou, ${ }^{55,43}$ Xiaoyu Zhou, ${ }^{20}$ Xu Zhou, ${ }^{20}$ A. N. Zhu, ${ }^{1,47}$ J. Zhu, ${ }^{34}$ J. Zhu, ${ }^{44}$ K. Zhu, ${ }^{1}$ K. J. Zhu, ${ }^{1,43,47}$ S. H. Zhu, ${ }^{54}$ W. J. Zhu, ${ }^{34}$ X. L. Zhu, ${ }^{45}$ Y. C. Zhu, ${ }^{55,43}$ Y. S. Zhu, ${ }^{1,47}$ Z. A. Zhu, ${ }^{1,47}$ J. Zhuang, ${ }^{1,43}$ B. S. Zou, ${ }^{1}$ and J. H. Zou ${ }^{1}$

\title{
(BESIII Collaboration)
}

\author{
${ }^{1}$ Institute of High Energy Physics, Beijing 100049, People's Republic of China \\ ${ }^{2}$ Beihang University, Beijing 100191, People's Republic of China \\ ${ }^{3}$ Beijing Institute of Petrochemical Technology, Beijing 102617, People's Republic of China \\ ${ }^{4}$ Bochum Ruhr-University, D-44780 Bochum, Germany \\ ${ }^{5}$ Carnegie Mellon University, Pittsburgh, Pennsylvania 15213, USA \\ ${ }^{6}$ Central China Normal University, Wuhan 430079, People's Republic of China \\ ${ }^{7}$ China Center of Advanced Science and Technology, Beijing 100190, People's Republic of China \\ ${ }^{8}$ COMSATS University Islamabad, Lahore Campus, Defence Road, Off Raiwind Road, 54000 Lahore, Pakistan \\ ${ }^{9}$ Fudan University, Shanghai 200443, People's Republic of China \\ ${ }^{10}$ G.I. Budker Institute of Nuclear Physics SB RAS (BINP), Novosibirsk 630090, Russia \\ ${ }^{11}$ GSI Helmholtzcentre for Heavy Ion Research GmbH, D-64291 Darmstadt, Germany \\ ${ }^{12}$ Guangxi Normal University, Guilin 541004, People's Republic of China \\ ${ }^{13}$ Guangxi University, Nanning 530004, People's Republic of China \\ ${ }^{14}$ Hangzhou Normal University, Hangzhou 310036, People's Republic of China \\ ${ }^{15}$ Helmholtz Institute Mainz, Johann-Joachim-Becher-Weg 45, D-55099 Mainz, Germany \\ ${ }^{16}$ Henan Normal University, Xinxiang 453007, People's Republic of China \\ ${ }^{17}$ Henan University of Science and Technology, Luoyang 471003, People's Republic of China \\ ${ }^{18}$ Huangshan College, Huangshan 245000, People's Republic of China \\ ${ }^{19}$ Hunan Normal University, Changsha 410081, People's Republic of China \\ ${ }^{20}$ Hunan University, Changsha 410082, People's Republic of China \\ ${ }^{21}$ Indian Institute of Technology Madras, Chennai 600036, India \\ ${ }^{22}$ Indiana University, Bloomington, Indiana 47405, USA \\ ${ }^{23 a}$ INFN Laboratori Nazionali di Frascati, I-00044 Frascati, Italy \\ ${ }^{23 \mathrm{~b}}$ INFN and University of Perugia, I-06100 Perugia, Italy \\ ${ }^{24 a}$ INFN Sezione di Ferrara, I-44122 Ferrara, Italy \\ ${ }^{24 \mathrm{~b}}$ University of Ferrara, I-44122 Ferrara, Italy \\ ${ }^{25}$ Institute of Physics and Technology, Peace Ave. 54B, Ulaanbaatar 13330, Mongolia \\ ${ }^{26}$ Johannes Gutenberg University of Mainz, Johann-Joachim-Becher-Weg 45, D-55099 Mainz, Germany \\ ${ }^{27}$ Joint Institute for Nuclear Research, 141980 Dubna, Moscow region, Russia \\ ${ }^{28}$ Justus-Liebig-Universitaet Giessen, II. Physikalisches Institut, Heinrich-Buff-Ring 16, D-35392 Giessen, Germany \\ ${ }^{29}$ KVI-CART, University of Groningen, NL-9747 AA Groningen, The Netherlands \\ ${ }^{30}$ Lanzhou University, Lanzhou 730000, People's Republic of China \\ ${ }^{31}$ Liaoning University, Shenyang 110036, People's Republic of China \\ ${ }^{32}$ Nanjing Normal University, Nanjing 210023, People's Republic of China \\ ${ }^{33}$ Nanjing University, Nanjing 210093, People's Republic of China \\ ${ }^{34}$ Nankai University, Tianjin 300071, People's Republic of China \\ ${ }^{35}$ Peking University, Beijing 100871, People's Republic of China \\ ${ }^{36}$ Shandong Normal University, Jinan 250014, People's Republic of China \\ ${ }^{37}$ Shandong University, Jinan 250100, People's Republic of China \\ ${ }^{38}$ Shanghai Jiao Tong University, Shanghai 200240, People's Republic of China \\ ${ }^{39}$ Shanxi University, Taiyuan 030006, People's Republic of China \\ ${ }^{40}$ Sichuan University, Chengdu 610064, People's Republic of China \\ ${ }^{41}$ Soochow University, Suzhou 215006, People's Republic of China \\ ${ }^{42}$ Southeast University, Nanjing 211100, People's Republic of China \\ ${ }^{43}$ State Key Laboratory of Particle Detection and Electronics, Beijing 100049, Hefei 230026, People's Republic of China \\ ${ }^{44}$ Sun Yat-Sen University, Guangzhou 510275, People's Republic of China \\ ${ }^{45}$ Tsinghua University, Beijing 100084, People's Republic of China
}




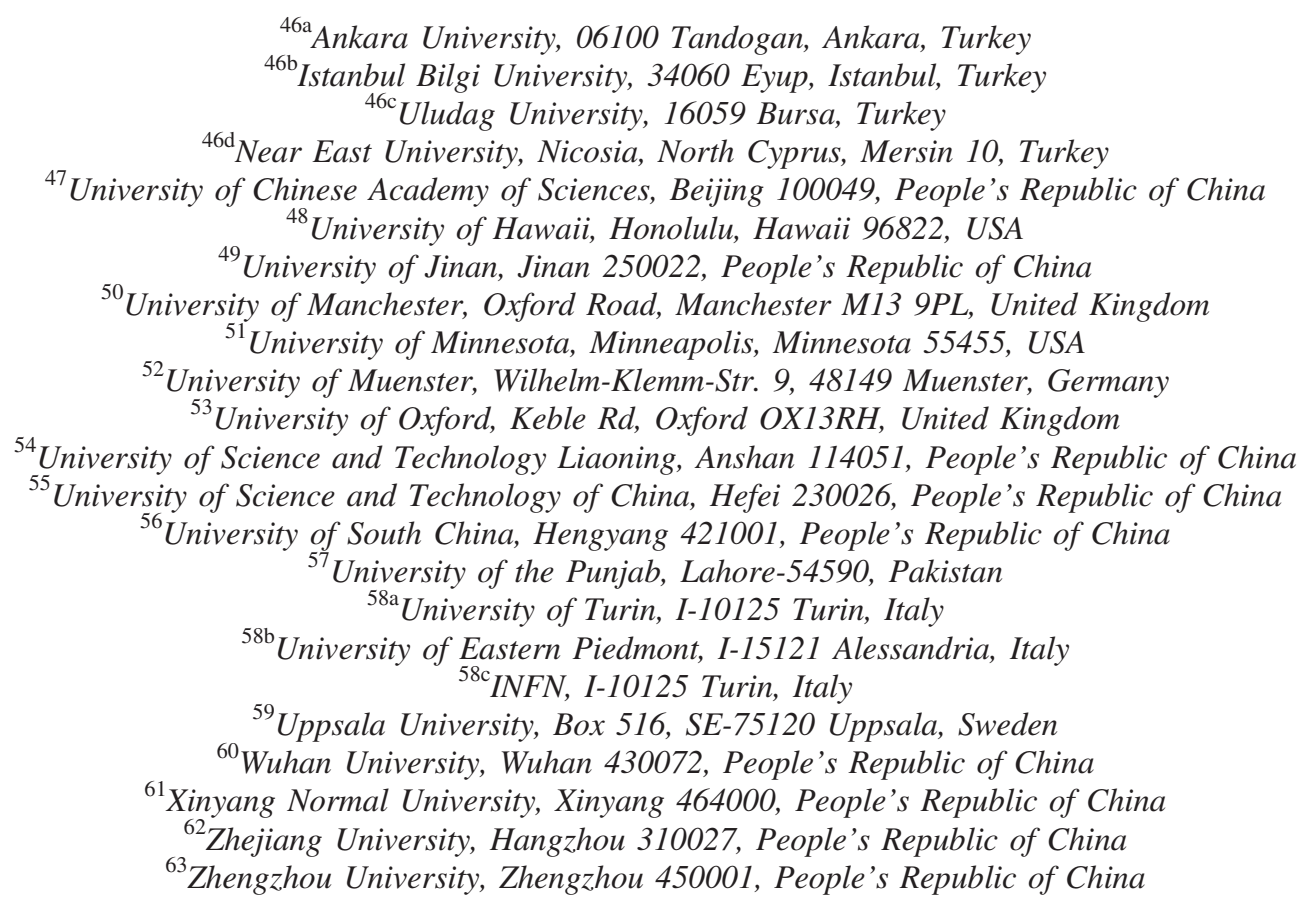

(Received 23 August 2019; published 22 November 2019)

We report the first observation of $D^{+} \rightarrow \tau^{+} \nu_{\tau}$ with a significance of 5.1 $\sigma$. We measure $\mathcal{B}\left(D^{+} \rightarrow \tau^{+} \nu_{\tau}\right)=$ $\left(1.20 \pm 0.24_{\text {stat }} \pm 0.12_{\text {syst }}\right) \times 10^{-3}$. Taking the world average $\mathcal{B}\left(D^{+} \rightarrow \mu^{+} \nu_{\mu}\right)=(3.74 \pm 0.17) \times 10^{-4}$, we obtain $R_{\tau / \mu}=\Gamma\left(D^{+} \rightarrow \tau^{+} \nu_{\tau}\right) / \Gamma\left(D^{+} \rightarrow \mu^{+} \nu_{\mu}\right)=3.21 \pm 0.64_{\text {stat }} \pm 0.43_{\text {syst }}$, which is consistent with the standard model expectation of lepton flavor universality. Using external inputs, our results give values for the $D^{+}$decay constant $f_{D^{+}}$and the Cabibbo-Kobayashi-Maskawa matrix element $\left|V_{c d}\right|$ that are consistent with, but less precise than, other determinations.

DOI: 10.1103/PhysRevLett.123.211802

In the purely leptonic decay of the charmed meson $D^{+}$, the $c$ and $\bar{d}$ quarks annihilate into a pair of charged and neutral leptons via a virtual $W$ boson. (Unless otherwise noted, charge conjugate modes are implied throughout this Letter.) To the lowest order, the decay rate for $D^{+} \rightarrow \ell^{+} \nu_{\ell}$ is given in a very simple form:

$\Gamma\left(D^{+} \rightarrow \ell^{+} \nu_{\ell}\right)=\frac{G_{F}^{2}}{8 \pi} f_{D^{+}}^{2}\left|V_{c d}\right|^{2} m_{\ell}^{2} M_{D^{+}}\left(1-\frac{m_{\ell}^{2}}{M_{D^{+}}^{2}}\right)^{2}$,

where the $D^{+}$mass $M_{D^{+}}$, the masses of the charged leptons $m_{\ell}\left(\ell=e^{+}, \mu^{+}\right.$, or $\left.\tau^{+}\right)$, and the Fermi coupling constant $G_{F}$ are known to great precision [1]. Because of this, measuring $\mathcal{B}\left(D^{+} \rightarrow \ell^{+} \nu_{\ell}\right)\left(\mathcal{B}_{\ell \nu}\right)$ allows determination of the product $f_{D^{+}}^{2}\left|V_{c d}\right|^{2}$ of the $D^{+}$decay constant and the

Published by the American Physical Society under the terms of the Creative Commons Attribution 4.0 International license. Further distribution of this work must maintain attribution to the author(s) and the published article's title, journal citation, and DOI. Funded by SCOAP. square of the $c \rightarrow d$ Cabibbo-Kobayashi-Maskawa (CKM) matrix element. One can then extract $\left|V_{c d}\right|$ by using the predicted value of $f_{D^{+}}$, e.g., from lattice quantum chromodynamics (LQCD), or obtain $f_{D^{+}}$by using the experimentally measured $\left|V_{c d}\right|$ to test the LQCD prediction. Such studies have been done using the muonic mode $D^{+} \rightarrow$ $\mu^{+} \nu_{\mu}$ ([2], [3]), which is a simple two-body decay with a clear experimental signature. The energetic track produced in this decay can be reconstructed very efficiently with minimal systematic uncertainty.

Experimental information about $D^{+} \rightarrow \tau^{+} \nu_{\tau}$ is more sparse, with only an upper limit of $1.2 \times 10^{-3}$ on $\mathcal{B}_{\tau \nu}$ at a $90 \%$ confidence level (C.L.) [1] that was set by the CLEO Collaboration [3]. Measuring $\mathcal{B}_{\tau \nu}$ is an important check of the standard model, which predicts the ratio of the $\tau^{+} \nu_{\tau}$ and $\mu^{+} \nu_{\mu}$ decay rates. Applying Eq. (1) to both $D^{+} \rightarrow \mu^{+} \nu_{\mu}$ and $D^{+} \rightarrow \tau^{+} \nu_{\tau}$, we find

$$
R_{\tau / \mu}=\frac{\Gamma\left(D^{+} \rightarrow \tau^{+} \nu_{\tau}\right)}{\Gamma\left(D^{+} \rightarrow \mu^{+} \nu_{\mu}\right)}=\frac{m_{\tau}^{2}\left(1-\frac{m_{\tau}^{2}}{M_{D^{+}}^{2}}\right)^{2}}{m_{\mu}^{2}\left(1-\frac{m_{\mu}^{2}}{M_{D^{+}}^{2}}\right)^{2}}=2.67,
$$


which provides a clean test of the standard model expectation of lepton flavor universality. Deviation from the expected value of $R_{\tau / \mu}$ could signify contributions of a charged intermediate boson that couples to the leptons differently, e.g., through a leptoquark [4]. The fact that $\mathcal{B}_{\tau \nu}$ has not been measured previously, together with the recent hints of possible violation of lepton universality in $B$ decays [5], establishes that $R_{\tau / \mu}$ is an important quantity to determine experimentally. We note, however, that in some supersymmetric models, such as the two-Higgs-doublet model [6], the charged Higgs couples to the lepton mass leading to a mass dependence identical to that from the $W$ boson process, including its helicity suppression. Thus, Eq. (1) is modified by a factor that does not depend on the lepton masses, leaving $R_{\tau / \mu}$ unchanged.

From the standard model prediction $R_{\tau / \mu}=2.67$ and $\mathcal{B}_{\mu \nu}=(3.74 \pm 0.17) \times 10^{-4} \quad[1], \quad$ one expects $\quad \mathcal{B}_{\tau \nu}=$ $(1.01 \pm 0.05) \times 10^{-3}$, which is very close to CLEO's upper limit based on $818 \mathrm{pb}^{-1}$ of $e^{+} e^{-}$annihilation data. In this Letter, we report the first observation of $D^{+} \rightarrow \tau^{+} \nu_{\tau}$ and the measurement of its branching fraction with an $e^{+} e^{-}$ annihilation sample produced at the Beijing Electron Positron Collider (BEPCII) [7] near the nominal mass of the $\psi(3770)$ resonance, $\sqrt{s}=3.773 \mathrm{GeV}$, with an integrated luminosity of $2931.8 \mathrm{pb}^{-1}$ [8] collected with the BESIII detector

BESIII is a cylindrical detector with a solid angle coverage of $93 \%$ of $4 \pi$. The detector consists of a Helium-gas-based main drift chamber (MDC), a plastic scintillator time-of-flight system, a CsI(Tl) electromagnetic calorimeter (EMC), a superconducting solenoid providing a $1.0 \mathrm{~T}$ magnetic field, and muon counters. The charged particle momentum resolution is $0.5 \%$ at a transverse momentum of $1 \mathrm{GeV} / c$. The photon energy resolution at $1 \mathrm{GeV}$ is $2.5 \%$ in the central barrel region and $5.0 \%$ in the end cap region. More details about the design and performance of BESIII are given in Ref. [9].

Detection efficiencies and background processes are determined with a Monte Carlo (MC) simulation sample with an equivalent luminosity roughly 10 times larger than the data set. It consists of events from $e^{+} e^{-} \rightarrow \psi(3770) \rightarrow$ $D \bar{D}, \quad e^{+} e^{-} \rightarrow q \bar{q} \quad(q=u, d, s), \quad e^{+} e^{-} \rightarrow \gamma J / \psi, \quad e^{+} e^{-} \rightarrow$ $\gamma \psi(3686)$, and $e^{+} e^{-} \rightarrow \tau^{+} \tau^{-}$. The effects of initial- and final-state radiation are simulated by the KKMC generator [10] and the PHOTOS package [11], respectively. The generated four-momenta are propagated into EVTGEN [12], which simulates decays using known rates [13] and correct angular distributions. We generate charmonium decays not accounted for by exclusive measurements with LUNDCHARM [14]. Finally, the detector response is simulated with GEANT4 [15].

We measure $\mathcal{B}_{\tau \nu}$ by reconstructing $\tau^{+}$via $\tau^{+} \rightarrow \pi^{+} \bar{\nu}_{\tau}$, which has the feature of only a single charged track from the $D^{+}$decay. Because pions and muons are charged particles with similar masses, the BESIII selection of pion tracks based on specific-ionization and time-of-flight measurements also accepts muon tracks with comparable efficiency (>90\%), allowing simultaneous measurement of $\mathcal{B}_{\tau \nu}$ and $\mathcal{B}_{\mu \nu}$. For this analysis our main result is obtained by fixing $\mathcal{B}_{\mu \nu}$ to the world average of $(3.74 \pm 0.17) \times 10^{-4}$ [1] to maximize our statistical sensitivity for measuring $\mathcal{B}_{\tau \nu}$. We also perform a cross-check of our result by measuring $\mathcal{B}_{\mu \nu}$ and $\mathcal{B}_{\tau \nu}$ simultaneously.

This analysis employs a double-tag technique, pioneered by the Mark III Collaboration [16]. We obtain the branching fraction by reconstructing $D^{+} \rightarrow \tau^{+}\left(\rightarrow \pi^{+} \bar{\nu}_{\tau}\right) \nu_{\tau}$ in events with $D^{-}$decays reconstructed in one of the six tag modes listed in Table I:

$$
\mathcal{B}_{\tau \nu}=\frac{N_{\tau \nu}}{\sum_{i} N_{\text {tag }}^{i}\left(\epsilon_{\tau \nu}^{i} / \epsilon_{\text {tag }}^{i}\right)} .
$$

In Eq. (3) $N_{\tau \nu}$ is the number of events with any $D^{-}$tag and a $D^{+} \rightarrow \tau^{+}\left(\rightarrow \pi^{+} \bar{\nu}_{\tau}\right) \nu_{\tau}$ candidate, $\epsilon_{\tau \nu}^{i}$ is the signal selection efficiency including $\mathcal{B}\left(\tau^{+} \rightarrow \pi^{+} \bar{\nu}_{\tau}\right)$ for an event with a $D^{-}$ in the $i$ th tag mode, and $N_{\text {tag }}^{i}$ and $\epsilon_{\text {tag }}^{i}$ are the number of tag and reconstruction efficiency for $D^{-}$tags in mode $i$.

In selecting tags our criteria for the final-state particles are identical to those in Ref. [17]. In each event, we allow only one $D$ candidate for a given tag mode separately for $D^{+}$and $D^{-}$, following the method of Ref. [18]. For each tag mode, we extract $N_{\text {tag }}^{i}$ from distributions of beamconstrained mass $M_{\mathrm{BC}} c^{2}=\sqrt{E_{\text {beam }}^{2}-\left|\vec{p}_{\text {tag }} c\right|^{2}}$, where $\vec{p}_{\text {tag }}$ is the three-momentum of the $\operatorname{tag} D^{-}$candidate and $E_{\text {beam }}$ is the beam energy in the center-of-mass system of the $\psi(3770)$. We fit to these $M_{\mathrm{BC}}$ distributions with MC-based signal shapes that are convolved with a Gaussian to accommodate resolution differences between simulation and data. The background shape is parametrized with an ARGUS function [19]. Figure 1 shows the fits to $M_{\mathrm{BC}}$ distributions. To select the tag, we require that $1863<$ $M_{\mathrm{BC}}<1877 \mathrm{MeV} / c^{2}$ [20]. Table I shows $N_{\mathrm{tag}}^{i}, \epsilon_{\mathrm{tag}}^{i}$, and $\epsilon_{\tau \nu}^{i}$ for all tag modes.

Once we select the tag, we require that there be only one additional charged track and that it have charge opposite

TABLE I. Single-tag efficiencies $\left(\epsilon_{\mathrm{tag}}^{i}\right)$ and yields $\left(N_{\mathrm{tag}}^{i}\right)$, and signal selection efficiencies $\left(\epsilon_{\tau \nu}^{i}\right)$. Efficiencies are corrected for $\mathcal{B}\left(K_{S}^{0} \rightarrow \pi^{+} \pi^{-}\right)$and $\mathcal{B}\left(\pi^{0} \rightarrow \gamma \gamma\right)$.

\begin{tabular}{lrcc}
\hline \hline Tag modes, $i$ & $N_{\mathrm{tag}}^{i}\left(\times 10^{3}\right)$ & $\epsilon_{\mathrm{tag}}^{i}(\%)$ & $\epsilon_{\tau \nu}^{i}(\%)$ \\
\hline$K^{+} \pi^{-} \pi^{-}$ & $797.6 \pm 1.0$ & $51.06 \pm 0.03$ & $3.6 \pm 0.1$ \\
$K^{+} \pi^{-} \pi^{-} \pi^{0}$ & $245.1 \pm 0.7$ & $25.18 \pm 0.03$ & $2.1 \pm 0.1$ \\
$K_{S}^{0} \pi^{-}$ & $92.6 \pm 0.3$ & $50.66 \pm 0.07$ & $4.0 \pm 0.1$ \\
$K_{S}^{0} \pi^{-} \pi^{0}$ & $206.3 \pm 0.6$ & $26.09 \pm 0.03$ & $2.1 \pm 0.1$ \\
$K_{S}^{0} \pi^{-} \pi^{-} \pi^{+}$ & $110.2 \pm 0.4$ & $26.75 \pm 0.04$ & $2.2 \pm 0.1$ \\
$K^{+} K^{-} \pi^{-}$ & $68.1 \pm 0.3$ & $40.38 \pm 0.08$ & $3.1 \pm 0.1$ \\
\hline \hline
\end{tabular}




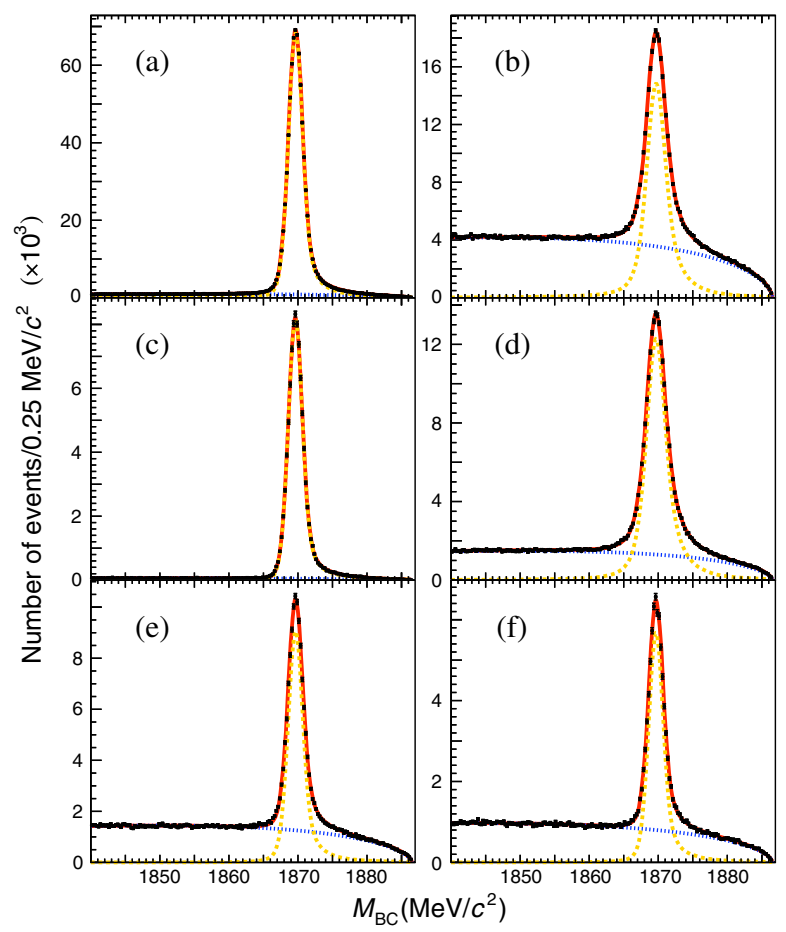

FIG. 1. Fits to $M_{\mathrm{BC}}$ distributions of single-tag $D^{-}$candidates for the full data sample for tag modes $D^{-} \rightarrow$ (a) $K^{+} \pi^{-} \pi^{-}$, (b) $K^{+} \pi^{-} \pi^{-} \pi^{0}$, (c) $K_{S}^{0} \pi^{-}$, (d) $K_{S}^{0} \pi^{-} \pi^{0}$, (e) $K_{S}^{0} \pi^{-} \pi^{-} \pi^{+}$, and (f) $K^{+} K^{-} \pi^{-}$. Red lines are the overall fits, while the yellowdashed (blue-dotted) lines are the fitted signals (backgrounds).

to that of the tag. It must originate within $1 \mathrm{~cm}(10 \mathrm{~cm})$ from the beam interaction point in the plane transverse to (along) the beam direction, be within the fiducial region for reliable track reconstruction $(|\cos \theta|<0.93$, where $\theta$ is the polar angle with respect to the direction of the positron beam), and match a shower in the EMC. Furthermore, to distinguish between $\pi$-like and $\mu$-like tracks, we rely on the minimum-ionizing character of the $\mu$ track, which has a mean energy deposit of $E_{\mathrm{EMC}} \simeq 200 \mathrm{MeV}$, as was done in Refs. [2,3]. Thus we partition the selected events into two samples, one with $\mu$-like tracks $\left(E_{\mathrm{EMC}} \leq 300 \mathrm{MeV}\right)$ and the other with $\pi$-like tracks $\left(E_{\mathrm{EMC}}>300 \mathrm{MeV}\right)$. The first portion includes $99 \%$ of the muon tracks from $D^{+} \rightarrow \mu^{+} \nu_{\mu}$, while the second has $44 \%$ of the pion tracks from $D^{+} \rightarrow \tau^{+} \nu_{\tau}, \tau^{+} \rightarrow \pi^{+} \bar{\nu}_{\tau}$.

To suppress backgrounds further, we apply four additional requirements, which are optimized based on $\mathrm{MC}$ calculations. (1) $E_{\mathrm{EMC}} /|\vec{p} c|<0.95$ for the $\pi$-like sample, where $\vec{p}$ is the signal track momentum measured by the MDC. As this variable sharply peaks around 1 for an electron, this requirement suppresses events from semileptonic decays like $D^{+} \rightarrow K_{L}^{0} e^{+} \nu_{e}$. (2) $E_{\max }<300 \mathrm{MeV}$ for both samples, where $E_{\max }$ is the maximum energy of all EMC showers that are not assigned to any charged track or neutral EMC shower in the reconstruction of both $D^{+}$and $D^{-}$. This suppresses events with extra particles, including misreconstructed neutral pions. (3) $\left|\cos \theta_{\text {missing }}\right|<$ $0.95(0.75)$ for the $\mu(\pi)$-like sample, where $\theta_{\text {missing }}$ is the polar angle of the missing momentum $\vec{p}_{\text {missing }}=-\vec{p}_{D^{-}}-$ $\vec{p}_{\mu(\pi)}, \vec{p}_{D^{-}}=\hat{p}_{D^{-}} \sqrt{\left(E_{\text {beam }} / c\right)^{2}-\left(M_{D^{+}} c\right)^{2}}$, and $\hat{p}_{D^{-}}$is the unit momentum vector of the $D^{-}$. This ensures that $\vec{p}_{\text {missing }}$ points to an active region of the detector. (4) $\alpha>25^{\circ}\left(45^{\circ}\right)$ for the $\mu(\pi)$-like sample, where $\alpha$ is the opening angle between $\vec{p}_{\text {missing }}$ and the direction of the most energetic unassigned shower. A shower from an asymmetric decay of $\pi^{0}$ or from $K_{L}^{0}$ tends to deposit energy in the EMC in the $\vec{p}_{\text {missing }}$ direction. The minimum required energy of the unassigned shower is $25 \mathrm{MeV}$ for $|\cos \theta|<0.8$ and $50 \mathrm{MeV}$ for $0.86<|\cos \theta|<0.93$.

Signals are extracted from the distributions of missing mass-squared $M_{\text {miss }}^{2}=E_{\text {missing }}^{2}-\left|\vec{p}_{\text {missing }} c\right|^{2}$, where $E_{\text {missing }}=$ $E_{\text {beam }}-E_{\mu(\pi)}$. Events from $D^{+} \rightarrow \mu^{+} \nu_{\mu}$ peak around $M_{\text {miss }}^{2}=0$, and the ones from $D^{+} \rightarrow \tau^{+} \nu_{\tau}$, where $\tau^{+} \rightarrow$ $\pi^{+} \bar{\nu}_{\tau}$, also tend to populate near $M_{\text {miss }}^{2}=0$ because $m_{\tau} \simeq M_{D}$.

We expect peaking backgrounds from $D^{+} \rightarrow \pi^{0} \pi^{+}$and $D^{+} \rightarrow K_{L}^{0} \pi^{+}$. The first is relatively small, but is close to $M_{\text {miss }}^{2}=0$. The latter peaks away from $M_{\text {miss }}^{2}=0$ at $\mathrm{m}_{K^{0}}^{2}$, but is a concern because of an expected rate of 40 times the expected signal.

We use data-based control samples to construct the probability density functions (PDFs) to represent these two peaking backgrounds. The black points in Fig. 2 show $M_{\text {miss }}^{2}$ distributions from exclusively reconstructed $D^{+} \rightarrow \pi^{0}(\rightarrow \gamma \gamma) \pi^{+} \quad$ (left column) and $D^{+} \rightarrow K_{S}^{0}(\rightarrow$ $\left.\pi^{+} \pi^{-}\right) \pi^{+}$(right column) events in which we treat the $K_{S}^{0}$ and the $\pi^{0}$ as missing particles, respectively. The redshaded histograms are from true $D^{+} \rightarrow \pi^{0} \pi^{+}$and $D^{+} \rightarrow$ $K_{L}^{0} \pi^{+} \mathrm{MC}$ events after applying all signal selection criteria, scaled to the same sizes as the data. Agreement between the shapes of the expected distributions and our control samples is excellent. We generate the corresponding PDFs by smoothing the distributions of the data points by the kernel estimation method [21]. Additional peaking backgrounds from $D^{+} \rightarrow \eta(\rightarrow \gamma \gamma) \pi^{+}$and $D^{+} \rightarrow K_{S}^{0}\left(\rightarrow \pi^{0} \pi^{0}\right) \pi^{+}$ are also considered, but both are small and peak away from $M_{\text {miss }}^{2}=0$. For these two small backgrounds, we use the MC events to predict the PDF.

We perform an unbinned simultaneous maximum likelihood fit to the $\mu$ - and $\pi$-like samples. The signal PDFs are based on MC events, including $D^{+} \rightarrow \tau^{+} \nu_{\tau}$ with $\tau^{+}$final states other than $\pi^{+} \bar{\nu}_{\tau}$. This contribution is dominated by $\tau^{+} \rightarrow \mu^{+} \nu_{\mu} \bar{\nu}_{\tau}$ and $\pi^{+} \pi^{0} \bar{\nu}_{\tau}$ in the $\mu$-like sample, while the $\pi$-like sample mostly contains $\tau^{+} \rightarrow e^{+} \nu_{e} \bar{\nu}_{\tau}$ and $\pi^{+} \pi^{0} \bar{\nu}_{\tau}$. To take into account the $M_{\text {miss }}^{2}$ resolution difference between the data and the MC samples, the PDFs of the signal and of the backgrounds are smeared using a Gaussian. The Gaussian mean and width are free parameters of the fit. The remaining background ("smooth background") comes 


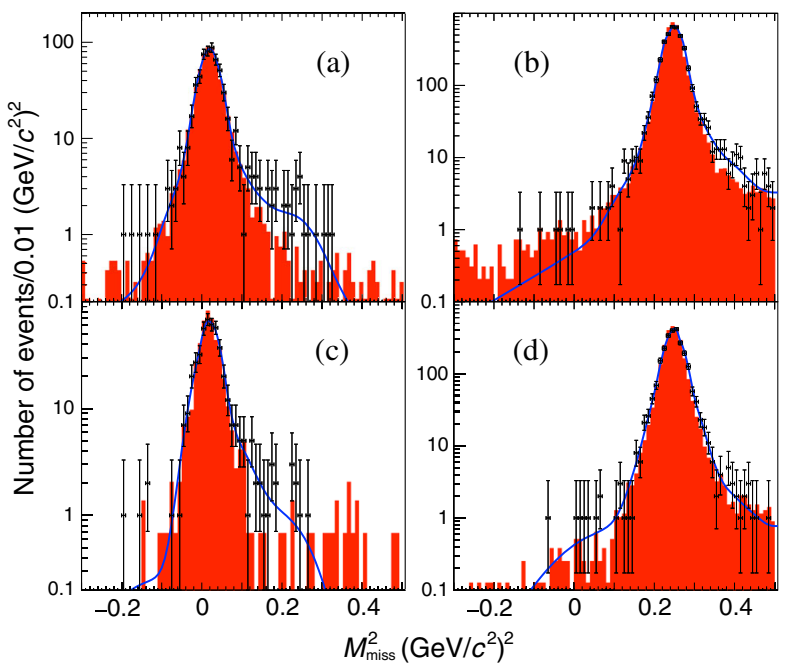

FIG. 2. $M_{\text {miss }}^{2}$ distributions of $D^{+} \rightarrow \pi^{0} \pi^{+}$(a), (c) and $D^{+} \rightarrow$ $K_{S}^{0}\left(\rightarrow \pi^{+} \pi^{-}\right) \pi^{+}$(b), (d) events from data (black points) for the $\mu$-like (a), (b) and $\pi$-like (c), (d) samples. The blue lines are the PDFs derived from the black points, while the red-shaded histograms are true $D^{+} \rightarrow \pi^{0} \pi^{+}$and $D^{+} \rightarrow K_{L}^{0} \pi^{+}$MC events with all selection criteria applied.

from other well known $D$ decays, such as semileptonic decays, as well as continuum events. It is represented by the smoothed MC prediction. We fix the sizes of $D^{+}$decays to $\mu^{+} \nu_{\mu}, \pi^{0} \pi^{+}, \eta \pi^{+}$, and $K_{S}^{0} \pi^{+}$according to Ref. [1], while we leave the normalizations for decays to $\tau^{+} \nu_{\tau}$ and $K_{L}^{0} \pi^{+}$, as well as the smooth background as free fit parameters. The ratio of the normalizations of the smooth background between the $\mu$-like and $\pi$-like samples is constrained based on the $\mathrm{MC}$ prediction. Applying this fitting procedure to the
$D \bar{D}$ MC sample, we obtain the signal selection efficiencies $\epsilon_{\tau \nu}^{i}$ for each tag mode listed in Table I.

Figure 3 shows the simultaneous fit to data, which yields $137 \pm 27$ signal events. This corresponds to $\mathcal{B}_{\tau \nu}=$ $\left(1.20 \pm 0.24_{\text {stat }}\right) \times 10^{-3}$.

As a cross check, we treat the $D^{+} \rightarrow \mu^{+} \nu_{\mu}$ component as a free fit parameter and obtain $\mathcal{B}_{\mu \nu}=\left(3.70 \pm 0.20_{\text {stat }}\right) \times$ $10^{-4}$, along with $\mathcal{B}_{\tau \nu}=\left(1.21 \pm 0.24_{\text {stat }}\right) \times 10^{-3}$. The obtained $\mathcal{B}_{\mu \nu}$ is consistent with both the world average of $(3.74 \pm 0.17) \times 10^{-4}$ [1] and the recent BESIII measurement of $\left(3.71 \pm 0.19_{\text {stat }} \pm 0.06_{\text {syst }}\right) \times 10^{-4}$ [2]. The agreement with the latter measurement provides independent confirmation, as Ref. [2] uses muon counter information and is based on simulations of the signal efficiency and the background that are different from the current work.

The total systematic uncertainty is dominated by two sources. The first is the uncertainty on $\mathcal{B}_{\mu \nu}$, which is fixed to the value from Ref. [1]. The second is the uncertainty due to the assumed shapes of the smooth background. For this we vary the shape by changing the $e^{+} e^{-} \rightarrow \psi(3770) \rightarrow D \bar{D}$ and $e^{+} e^{-} \rightarrow q \bar{q}$ cross sections from the defaults in our MC calculations. We also consider two different values of the smoothing parameter $\rho$ in the Gaussian kernel estimation method [21], $\rho=1$ (the author's suggestion) and $\rho=2$. The dependence on the choice of $300 \mathrm{MeV}$ for the boundary between $\pi$ - and $\mu$-like samples, which potentially changes the shapes of the smooth backgrounds, is also assessed by varying it by $\pm 50 \mathrm{MeV}$.

Other sources of systematic uncertainty are also considered. The uncertainty in the signal track reconstruction efficiency has been obtained from previous BESIII studies of double-tagged $D \bar{D}$ events. The uncertainty in
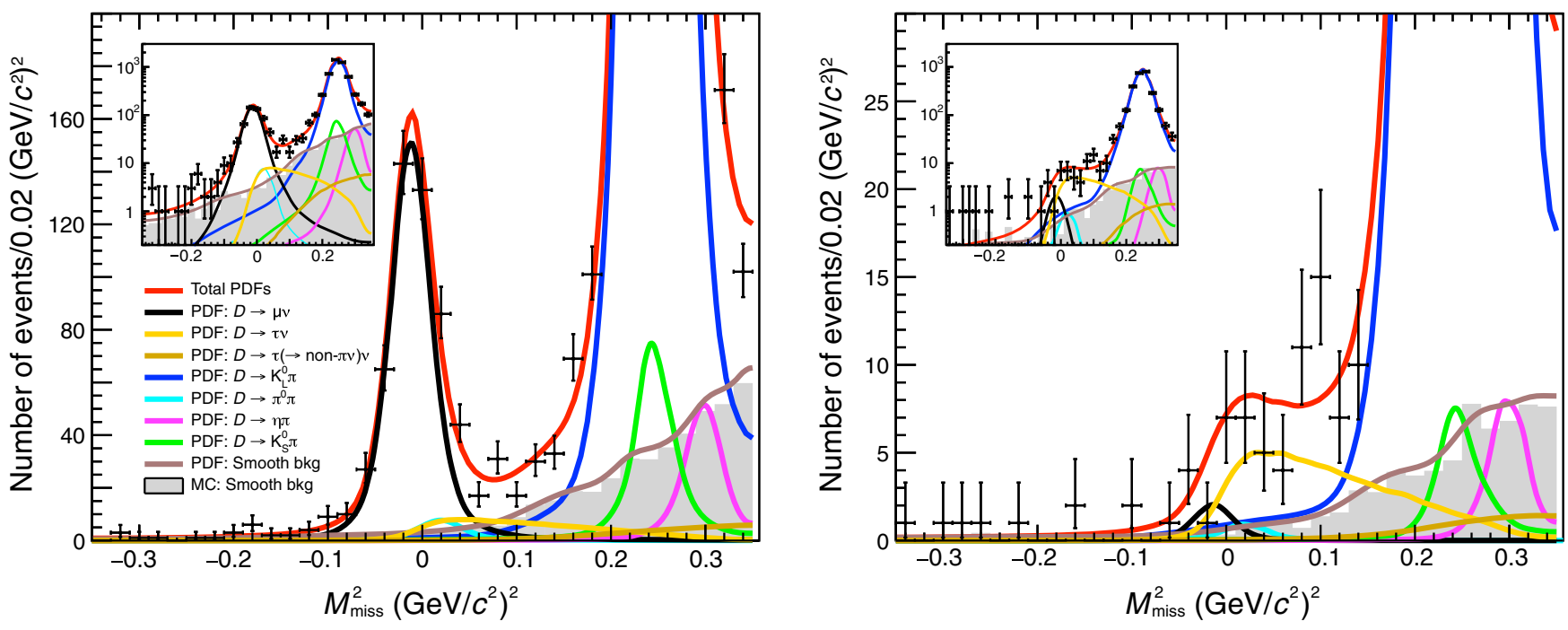

FIG. 3. Fits to $M_{\text {miss }}^{2}$ distributions of the $\mu$-like (left) and $\pi$-like (right) samples. The black points are data and gray-shaded histograms are MC-predicted smooth background components scaled to the data size based on the known production cross sections and measured integrated luminosity. The insets show the same distributions with logarithmic scales. 
TABLE II. Summary of relative systematic uncertainties in units of $10^{-2}$.

\begin{tabular}{lc}
\hline \hline Source & $\Delta \mathcal{B}_{\tau \nu}$ \\
\hline$\Delta \mathcal{B}\left(D^{+} \rightarrow \mu^{+} \nu_{\mu}\right)$ & 6.9 \\
Shape of smooth background & 4.2 \\
$\pi^{+}$tracking & 1.0 \\
$\Delta \mathcal{B}\left(\tau^{+} \rightarrow \pi^{+} \bar{\nu}_{\tau}\right)$ & 0.5 \\
Stat. uncertainties from tag side and MC calculations & 2.2 \\
Fitting scheme on tag side & 0.5 \\
Requirement on $E_{\mathrm{EMC}} /|\vec{p} c|$ & 2.5 \\
Requirement on $E_{\max }$ & 2.2 \\
Requirements on $\left|\cos \theta_{\text {missing }}\right|$ and $\alpha$ & 2.1 \\
Tag bias & 0.1 \\
Normalizations of small peaking backgrounds & 1.9 \\
Relative size of smooth background components & 2.5 \\
Signal shape of $D^{+} \rightarrow \tau^{+} \nu_{\tau}$ & 1.1 \\
Total systematic uncertainty & 9.9 \\
\hline \hline
\end{tabular}

$\mathcal{B}\left(\tau^{+} \rightarrow \pi^{+} \bar{\nu}_{\tau}\right)$ is from Ref. [1]. Statistical uncertainties in the tag counts in data and MC calculations are taken directly from the respective samples. Variations in the fit ranges, selection windows, binning, and signal and background parametrizations are used to probe uncertainties in the tag-side fits. We estimate uncertainties due to the $E_{\mathrm{EMC}} /|\vec{p} c|$ and $E_{\max }$ criteria with double-tagged events including $D^{+} \rightarrow K_{S}^{0} \pi^{+}$. Uncertainties from the cuts on $\left|\cos \theta_{\text {missing }}\right|$ and $\alpha$ are estimated with fully reconstructed $D^{0} \rightarrow K^{-} e^{+} \nu_{e}$ events. Possible mismodeling of efficiencies due to multiplicity differences among $D$ decay modes is estimated based on a study of tracking and particle identification efficiencies in different event environments. The uncertainty due to the normalization of the peaking backgrounds, and the ratio of smooth background sizes between $\mu$ - and $\pi$-like samples in the $M_{\text {miss }}^{2}$ fit are estimated by studies of the $D^{+} \rightarrow K_{S}^{0} \pi^{+}$control sample and by varying parametrizations and branching fractions, respectively. The $D^{+} \rightarrow \tau^{+} \nu_{\tau}$ signal-shape dependence is estimated by altering the mixture of $\tau^{+}$decay modes. Table II summarizes the systematic uncertainty estimate.

Using the $2.93 \mathrm{fb}^{-1}$ data sample taken at $\sqrt{s}=$ $3.773 \mathrm{GeV}$, we measure $\mathcal{B}_{\tau \nu}=\left(1.20 \pm 0.24_{\text {stat }} \pm 0.12_{\text {syst }}\right) \times$ $10^{-3}$ using $\mathcal{B}_{\mu \nu}=(3.74 \pm 0.17) \times 10^{-4}$. The signal significance including the systematic uncertainty is $5.1 \sigma$, calculated via $\sqrt{-2 \times \ln \mathcal{L}_{\text {null }} / \mathcal{L}}$, where $\mathcal{L}_{\text {null }}$ and $\mathcal{L}$ are likelihood values without and with $D^{+} \rightarrow \tau^{+} \nu_{\tau}$, respectively. This is the first measurement of the branching fraction of $D^{+} \rightarrow \tau^{+} \nu_{\tau}$ to date. With $\mathcal{B}_{\mu \nu}=(3.74 \pm 0.17) \times$ $10^{-4}$ [1], we find $R_{\tau / \mu}=3.21 \pm 0.64_{\text {stat }} \pm 0.43_{\text {syst }}$, which is consistent with the standard model prediction of 2.67 . From Eq. (1), with the inputs shown in Table III and assuming $\left|V_{c d}\right|=0.22438 \pm 0.00044$ from the global fit [1], we obtain
TABLE III. External input parameters with uncertainties from Ref. [1].

\begin{tabular}{ll}
\hline \hline Parameter & \multicolumn{1}{c}{ Value } \\
\hline$m_{\mu}$ & $0.1056583745(24) \mathrm{GeV}$ \\
$m_{\tau}$ & $1.77686(12) \mathrm{GeV}$ \\
$M_{D^{+}}$ & $1.86965(5) \mathrm{GeV}$ \\
$\tau_{D^{+}}$ & $1.040(7) \mathrm{ps}$ \\
$G_{F}$ & $1.1663787(6) \times 10^{-5} \mathrm{GeV}^{-2}$ \\
\hline \hline
\end{tabular}

$$
f_{D^{+}}=224.5 \pm 22.8_{\text {stat }} \pm 11.3_{\text {syst }} \pm 0.9_{\text {ext-syst }} \mathrm{MeV},
$$

where the last uncertainty is due to external input parameters. This is consistent with the average between the recent four-flavor LQCD predictions of Refs. [22,23], $f_{D^{+}}=212.6 \pm 0.6 \mathrm{MeV}$, as well as with the experimental results for $D^{+} \rightarrow \mu^{+} \nu_{\mu}$ from the BESIII [2] and the CLEO [3] Collaborations.

Taking the average prediction for $f_{D^{+}}$from [22] and [23], we find

$$
\left|V_{c d}\right|=0.237 \pm 0.024_{\text {stat }} \pm 0.012_{\text {syst }} \pm 0.001_{\text {ex-syst }} .
$$

This is consistent with both the world average $\left|V_{c d}\right|=$ $0.218 \pm 0.004$ [1] and the global fit result [1].

The BESIII collaboration thanks the staff of BEPCII and the IHEP computing center for their strong support. This work is supported in part by National Key Basic Research Program of China under Contract No. 2015CB856700; National Natural Science Foundation of China (NSFC) under Contracts No. 11625523, No. 11635010, and No. 11735014; National Natural Science Foundation of China (NSFC) under Contract No. 11835012; the Chinese Academy of Sciences (CAS) Large-Scale Scientific Facility Program; Joint Large-Scale Scientific Facility Funds of the NSFC and CAS under Contracts No. U1532257, No. U1532258, No. U1732263, and No. U1832207; CAS Key Research Program of Frontier Sciences under Contracts No. QYZDJ-SSW-SLH003 and No. QYZDJSSW-SLH040; 100 Talents Program of CAS; INPAC and Shanghai Key Laboratory for Particle Physics and Cosmology; German Research Foundation DFG under Contract No. Collaborative Research Center CRC 1044, FOR 2359; Istituto Nazionale di Fisica Nucleare, Italy; Koninklijke Nederlandse Akademie van Wetenschappen (KNAW) under Contract No. 530-4CDP03; Ministry of Development of Turkey under Contract No. DPT2006K120470; National Science and Technology fund; The Knut and Alice Wallenberg Foundation (Sweden) under Contract No. 2016.0157; The Royal Society, UK under Contract No. DH160214; The Swedish Research Council; U.S. Department of Energy under Contracts No. DE-FG02-05ER41374, No. DE-SC-0010118, and 
No. DE-SC-0012069; and University of Groningen (RuG) and the Helmholtzzentrum für Schwerionenforschung GmbH (GSI), Darmstadt.

${ }^{a}$ Also at Bogazici University, 34342 Istanbul, Turkey.

${ }^{\mathrm{b}}$ Also at the Moscow Institute of Physics and Technology, Moscow 141700, Russia.

${ }^{c}$ Also at the Functional Electronics Laboratory, Tomsk State University, Tomsk 634050, Russia.

${ }^{\mathrm{d}}$ Also at the Novosibirsk State University, Novosibirsk 630090, Russia.

eAlso at the NRC "Kurchatov Institute," PNPI, Gatchina 188300, Russia.

${ }^{\mathrm{f}}$ Also at Istanbul Arel University, 34295 Istanbul, Turkey.

${ }^{\mathrm{g}}$ Also at Goethe University Frankfurt, 60323 Frankfurt am Main, Germany.

${ }^{\mathrm{h}}$ Also at Key Laboratory for Particle Physics, Astrophysics and Cosmology, Ministry of Education; Shanghai Key Laboratory for Particle Physics and Cosmology; Institute of Nuclear and Particle Physics, Shanghai 200240, People's Republic of China.

${ }^{i}$ Also at Government College Women University, Sialkot51310 Punjab, Pakistan.

${ }^{\mathrm{j}}$ Also at Key Laboratory of Nuclear Physics and Ion-beam Application (MOE) and Institute of Modern Physics, Fudan University, Shanghai 200443, People's Republic of China. ${ }^{k}$ Also at Harvard University, Department of Physics, Cambridge, Massachusetts 02138, USA.

[1] M. Tanabashi et al. (Particle Data Group), Phys. Rev. D 98, 030001 (2018).

[2] M. Ablikim et al. (BESIII Collaboration), Phys. Rev. D 89, 051104(R) (2014).

[3] B. I. Eisenstein et al. (CLEO Collaboration), Phys. Rev. D 78, 052003 (2008).

[4] B. A. Dobrescu and A. S. Kronfeld, Phys. Rev. Lett. 100, 241802 (2008).

[5] S. Bifani, S. Descotes-Genon, A. Romero Vidal, and M.-H. Schune, J. Phys. G 46, 023001 (2019); J. P. Lees et al. (BABAR Collaboration), Phys. Rev. Lett. 109, 101802 (2012); M. Huschle et al. (Belle Collaboration), Phys. Rev. D 92, 072014 (2015); R. Aaij et al. (LHCb Collaboration), Phys. Rev. Lett. 115, 111803 (2015); Y. Sato et al. (Belle Collaboration), Phys. Rev. D 94, 072007 (2016); S. Hirose et al. (Belle Collaboration), Phys. Rev. Lett. 118,
211801 (2017); R. Aaij et al. (LHCb Collaboration), Phys. Rev. Lett. 120, 171802 (2018); 113, 151601 (2014); J. High Energy Phys. 08 (2017) 055.

[6] A. G. Akeroyd and C. H. Chen, Phys. Rev. D 75, 075004 (2007).

[7] C. H. Yu et al., Proc. of International Particle Accelerator Conference (IPAC'16), Busan, Korea, 2016 (JACoW, Geneva, 2016), TUYA01.

[8] M. Ablikim et al. (BESIII Collaboration), Chin. Phys. C 37, 123001 (2013); Phys. Lett. B 753, 629 (2016).

[9] M. Ablikim et al. (BESIII Collaboration), Nucl. Instrum. Methods Phys. Res., Sect. A 614, 345 (2010).

[10] S. Jadach, B. F. L. Ward, and Z. Was, Phys. Rev. D 63, 113009 (2001).

[11] E. Barberio and Z. Was, Comput. Phys. Commun. 79, 291 (1994).

[12] D. J. Lange, Nucl. Instrum. Methods Phys. Res., Sect. A 462, 152 (2001); R. G. Ping, Chin. Phys. C 32, 599 (2008).

[13] K. Nakamura et al. (Particle Data Group), J. Phys. G 37, 075021 (2010) and 2011 partial update for the 2012 edition.

[14] J. C. Chen, G. S. Huang, X. R. Qi, D. H. Zhang, and Y. S. Zhu, Phys. Rev. D 62, 034003 (2000); Y. Rui-Ling, R.-G. Ping, and H. Chen, Chin. Phys. Lett. 31, 061301 (2014).

[15] S. Agostinelli et al., Nucl. Instrum. Methods Phys. Res., Sect. A 506, 250 (2003); J. Allison et al., IEEE Trans. Nucl. Sci. 53, 270 (2006); Z. Y. Deng et al., High Energy Phys. Nucl. Phys. 30, 371 (2006).

[16] R. M. Baltrusaitis et al. (MARK III Collaboration), Phys. Rev. Lett. 56, 2140 (1986); J. Adler et al. (MARK III Collaboration), Phys. Rev. Lett. 60, 89 (1988).

[17] M. Ablikim et al. (BESIII Collaboration), Phys. Rev. D 97, 072004 (2018).

[18] M. Ablikim et al. (BESIII Collaboration), Chin. Phys. C 42, 083001 (2018).

[19] H. Albrecht et al. (ARGUS Collaboration), Phys. Lett. B 241, 278 (1990).

[20] M. Ablikim et al. (BESIII Collaboration), Phys. Rev. D 94, 032001 (2016).

[21] K. S. Cranmer, Comput. Phys. Commun. 136, 198 (2001).

[22] N. Carrasco et al. (ETM Collaboration), Phys. Rev. D 91, 054507 (2015).

[23] A. Bazavov, C. Bernard, N. Brown, C. DeTar, A.X. El-Khadra et al. (Fermilab Lattice and MILC Collaborations), Phys. Rev. D 98, 074512 (2018). 